\title{
SPECT/CT and a Portable $\gamma$-Camera for Image-Guided Laparoscopic Sentinel Node Biopsy in Testicular Cancer
}

\author{
Oscar R. Brouwer ${ }^{1}$, Renato A. Valdés Olmos ${ }^{1}$, Lenka Vermeeren ${ }^{1}$, Cornelis A. Hoefnagel ${ }^{1}$, Omgo E. Nieweg ${ }^{2}$, \\ and Simon Horenblas ${ }^{3}$ \\ ${ }^{1}$ Department of Nuclear Medicine, The Netherlands Cancer Institute, Amsterdam, The Netherlands; ${ }^{2}$ Department of Surgery, The \\ Netherlands Cancer Institute, Amsterdam, The Netherlands; and ${ }^{3}$ Department of Urology, The Netherlands Cancer Institute, \\ Amsterdam, The Netherlands
}

\begin{abstract}
The purpose of this study was to evaluate the utility of SPECT/CT and real-time intraoperative imaging with a portable $\gamma$-camera for laparoscopic sentinel node (SN) localization in stage I testicular cancer. Methods: Ten patients with clinical stage I testicular cancer were studied between November 2006 and November 2010. Their mean age was 37 y (range, 25-50 y). The primary tumors were situated on the right side in 5 patients and on the left side in 5 . After a funicular block with $2 \%$ lidocaine, an average dose of $80 \mathrm{MBq}$ (range, 59-98 MBq) of ${ }^{99 m} \mathrm{Tc}-$ nanocolloid in a volume of $0.2 \mathrm{~mL}$ was injected into the testicular parenchyma. Shortly after injection, a 10-min dynamic study was performed, followed by the acquisition of static planar images at $15 \mathrm{~min}$ and $2 \mathrm{~h}$. SPECT/CT was performed at $2 \mathrm{~h}$. After image fusion, SNs were visualized, and their exact anatomic location was determined. The SPECT/CT images were displayed in the operation room to guide $\mathrm{SN}$ detection using a laparoscopic $\gamma$-ray probe and a portable $\gamma$-camera. Results: Lymphatic drainage to the retroperitoneum was seen in all patients. SPECT/CT identified interaortocaval or paracaval SNs in the 5 patients with right-sided tumors, one of whom had an additional SN adjacent to the testicular vessels. In all 5 patients with left-sided tumors, paraaortic SNs were visualized; a node along the testicular vessels was visualized in 2 of these 5 . Twenty-six SNs were laparoscopically removed (range, 1-4 per patient). An SN contained metastases in 1 case. No recurrences developed in the 9 patients with a tumorfree SN during a median follow-up of 21 mo (range, 2-50 mo). Conclusion: SPECT/CT enables accurate anatomic localization of retroperitoneal SNs in patients with testicular cancer, facilitating their laparoscopic retrieval. Real-time image guidance by a portable $\gamma$-camera improves intraoperative $\mathrm{SN}$ detection and appears to identify (20\%) additional SNs.
\end{abstract}

Key Words: testicular cancer; sentinel node; SPECT/CT; portable gamma camera

J Nucl Med 2011; 52:551-554

DOI: 10.2967/jnumed.110.086660

Received Dec. 15, 2010; revision accepted Jan. 31, 2011.

For correspondence contact: Oscar R. Brouwer, Department of Nuclear Medicine, The Netherlands Cancer Institute, Antoni van Leeuwenhoek Hospital, Plesmanlaan 121, 1066 CX, Amsterdam, The Netherlands.

E-mail: o.brouwer@nki.nl

COPYRIGHT $\odot 2011$ by the Society of Nuclear Medicine, Inc. he optimal management of regional lymph nodes in stage I testicular cancer remains controversial. A surveillance policy requires intensive, frequent follow-up visits, with costly examinations, and defers detection and treatment of lymph node metastases to a later stage. The established treatment options for nonseminomatous germ cell tumors consist of surveillance, retroperitoneal lymphadenectomy, or chemotherapy, resulting in overtreatment in 65\%-75\% of the patients $(1,2)$. Treatment options for seminomatous germ cell tumors at this stage are surveillance, radiotherapy, or chemotherapy. Here, overtreatment is even more frequent, because occult metastases are present in less than $20 \%$ of patients (2-5). Diagnostic techniques are needed that enable patients with lymph node dissemination to be treated at an early stage while preventing unnecessary treatment of those without dissemination. To this end, the sentinel node (SN) biopsy was introduced for patients with stage I testicular cancer using intratesticular radiocolloid administration (6). In this study, preoperative lymphatic mapping was performed using planar lymphoscintigraphy only. However, this technique solely provides 2-dimensional information, and exact preoperative anatomic SN localization was not possible. The introduction of hybrid SPECT/CT appears to solve this limitation. With the new generation of large-field $\gamma$-cameras, the functional information of SPECT can be combined with the morphologic data of CT by acquiring images in 1 session. This innovative approach enables accurate localization of retroperitoneal SNs (7).

Intraoperatively, the urologist localizes an $\mathrm{SN}$, guided by acoustic signals from the laparoscopic $\gamma$-ray probe. However, intraoperative spatial orientation using this device can be difficult, because a laparoscopic probe does not provide visual information. Recently, our initial experience with a portable $\gamma$-camera to provide real-time intraoperative visual guidance for laparoscopic SN localization in several urologic malignancies was reported (8). In the present study, our experience with SPECT/CT for preoperative SN localization combined with a portable $\gamma$-camera for imageguided laparoscopic SN biopsy in stage I testicular cancer patients is evaluated. 


\section{MATERIALS AND METHODS}

\section{Patients}

The current study involved 10 patients (mean age, $37 \mathrm{y}$; range, 25-50 y) with clinical stage I testicular cancer referred to The Netherlands Cancer Institute between November 2006 and November 2010. All patients had a testicular germ cell tumor, situated on the right side in 5 patients and on the left side in 5 (Table 1). Three patients had a past history of stage I testicular cancer on the contralateral side for which they had undergone orchidectomy without SN procedure or lymphadenectomy. Two of the patients (seminoma) had also received adjuvant radiotherapy, and a third patient (nonseminoma) had been treated with standard platinum-based chemotherapy because of elevated serum tumor markers during follow-up. The study protocol was approved by the Medical Ethical Committee, and all patients were included after giving informed consent.

\section{Preoperative Procedure}

Local anesthesia was obtained with a funicular block using $2 \%$ lidocaine, performed by the urologist in the outpatient clinic approximately $30 \mathrm{~min}$ before tracer administration. In the nuclear medicine department, an average dose of $80 \mathrm{MBq}$ (range, 59-98 MBq) of ${ }^{99 \mathrm{~m}} \mathrm{Tc}$-nanocolloid (GE Healthcare), in a volume of $0.2 \mathrm{~mL}$ with approximately $0.1 \mathrm{~mL}$ of air behind the solution to flush any remaining radioactivity in the syringe, was injected with a fine needle (25-gauge) into the affected testicular parenchyma, guided by palpation. Immediately after injection, anterior and lateral dynamic images were obtained with a dual-head $\gamma$-camera over $10 \mathrm{~min}$ to visualize the lymphatic flow and identify early draining lymph nodes. Subsequently, static planar images were acquired. Two hours after injection of the tracer, delayed planar images were obtained to differentiate first-echelon nodes from higher-echelon nodes and identify unexpected drainage patterns. In the same session, SPECT/CT was performed using a hybrid camera (Symbia T; Siemens). After correction for attenuation and scatter, the SPECT image was fused with the CT image and analyzed using 2-dimensional orthogonal reslicing. The first node in each nodal basin appearing on early planar imaging was con- sidered to be the SN. Nodes appearing later in the same basin were considered to be higher-echelon nodes. The level of an SN was marked on the skin to provide an external centering spot for the laser pointer of the portable $\gamma$-camera during the operation.

\section{Intraoperative Procedure}

All patients underwent SN excision and orchidectomy within $6 \mathrm{~h}$ after injection of the tracer. The SPECT/CT images were displayed in the operation room to guide the laparoscopic detection with a laparoscopic $\gamma$-ray probe (Europrobe; Euro Medical Instruments). In addition, SNs were intraoperatively identified using a portable $\gamma$-camera (Sentinella; Oncovision) in 9 patients. In the remaining patient, the portable $\gamma$-camera was not yet available. SN localization with the portable $\gamma$-camera was facilitated using a radioactive ${ }^{125} \mathrm{I}$ seed set on the tip of the laparoscopic probe, as previously described (8). This ${ }^{125}$ I seed, displayed separately (as a yellow circle) on the screen of the portable $\gamma$-camera, is used as a pointer. The camera could thus visualize the proximity of the probe to the ${ }^{99 \mathrm{~m}} \mathrm{Tc}$ signal and thereby provide visual guidance to the SN. Postexcision images were obtained with the portable $\gamma$-camera to provide certainty about the completeness of the surgical procedure. A hot node remaining after excision of an $\mathrm{SN}$ at the same location was considered part of a cluster of SNs and was also removed. All removed SNs were formalin-fixed, bisected, paraffin-embedded, and cut at a minimum of 6 levels at 50 - to $150-\mu \mathrm{m}$ intervals. Pathologic examination included hematoxylin and eosin staining and immunohistochemistry staining.

\section{RESULTS}

Intratesticular administration of $99 \mathrm{~m} \mathrm{Tc}-$ nanocolloid, under local anesthesia, proved to be easy to perform and was well tolerated. No side effects were observed. Lymphoscintigraphy in combination with SPECT/CT showed lymphatic drainage in all 10 patients $(100 \%)$, and a total of 21 SNs were preoperatively identified. SPECT/CT enabled the accurate localization of the SNs and provided anatomic reference points to plan

TABLE 1

Patient Characteristics and SN Results

\begin{tabular}{|c|c|c|c|c|c|c|c|c|}
\hline Patient & Age (y) & Side & Pathology & SPECT/CT findings & $\begin{array}{l}\text { Excised } \\
\text { nodes }\end{array}$ & $\begin{array}{c}\mathrm{SN} \\
\text { metastases }\end{array}$ & Recurrence & Follow-up (mo) \\
\hline 1 & 50 & Left & Seminoma & $1 \mathrm{SN}$, paraaortic zone & 1 & - & - & 50 \\
\hline 2 & 29 & Right & Seminoma & $\begin{array}{l}1 \mathrm{SN} \text {, paracaval zone } \\
1 \mathrm{SN} \text {, interaortocaval zone }\end{array}$ & 4 & - & - & 46 \\
\hline 3 & 34 & Right & Seminoma & 2 SNs, interaortocaval zone & 2 & - & - & 39 \\
\hline 4 & 33 & Left & Seminoma & 2 SNs, paraaortic zone & 4 & - & - & 39 \\
\hline 5 & 34 & Right & Nonseminoma & $\begin{array}{l}2 \mathrm{SNs} \text {, interaortocaval zone } \\
1 \mathrm{SN} \text {, right common iliac zone }\end{array}$ & 3 & - & - & 23 \\
\hline 6 & 41 & Left & Seminoma & $1 \mathrm{SN}$, paraaortic zone & 1 & - & - & 19 \\
\hline 7 & 48 & Right & Seminoma & $\begin{array}{l}2 \text { SNs, interaortocaval zone } \\
1 \mathrm{SN} \text {, adjacent to (right) } \\
\text { testicular vessels }\end{array}$ & 3 & + & - & 11 \\
\hline 8 & 37 & Right & Seminoma & 2 SNs, paracaval zone & 2 & - & - & 6 \\
\hline 9 & 35 & Left & Seminoma & $\begin{array}{l}1 \mathrm{SN} \text {, paraaortic zone } \\
1 \mathrm{SN} \text {, adjacent to (left) testicular } \\
\text { vessels }\end{array}$ & 3 & - & - & 6 \\
\hline 10 & 25 & Left & Nonseminoma & $\begin{array}{l}2 \mathrm{SNs} \text {, paraaortic zone } \\
1 \mathrm{SN} \text {, adjacent to (left) testicular } \\
\text { vessels }\end{array}$ & 3 & - & - & 2 \\
\hline
\end{tabular}



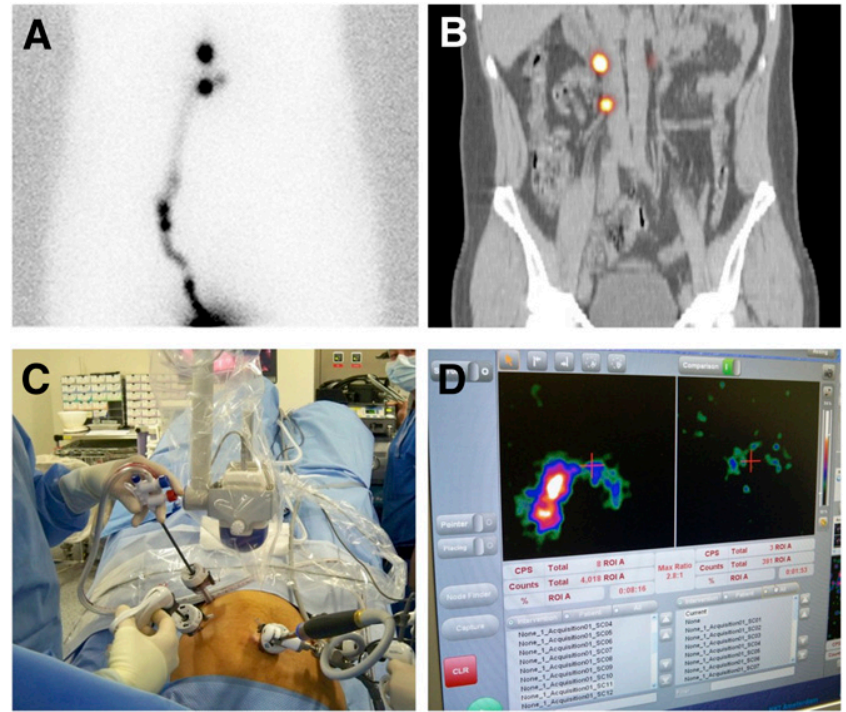

FIGURE 1. (A) Planar anterior image showing drainage of $99 \mathrm{mTC}-$ nanocolloid from injection site in right testicle to 2 abdominal lymph nodes and radioactivity along lymphatic channel, which decreased in time, indicating lymphatic tract visualization. (B) Coronal SPECT/ CT image fusion showing both sentinel lymph nodes (yellow) alongside inferior vena cava. (C) Laparoscopic SN procedure using portable $\gamma$-camera and laparoscopic $\gamma$-ray detection probe. (D) Preexcision image (left screen) acquired with portable $\gamma$-camera showing both $\mathrm{SNs}$ in operation room. After excision (right screen), no significant remaining activity is seen.

the laparoscopy in all patients. The 5 patients with right-sided tumors had SNs at interaortocaval or paracaval locations (Figs. 1 and 2). One of these patients also had an $\mathrm{SN}$ adjacent to the testicular vessels. All 5 patients with left-sided tumors had drainage to paraaortic SNs, and 2 of them had an additional $\mathrm{SN}$ along the testicular vessels as well (Fig. 3).

All preoperatively identified SNs were localized and excised (Table 1). Higher-echelon nodes were left in place. The portable $\gamma$-camera enabled real-time $\mathrm{SN}$ visualization in all 9 patients in whom the device was used $(100 \%)$. Moreover, intraoperative images after excision of an SN showed unexpected significant residual radioactivity at the same location in 3 of these patients, resulting in identification and removal of 5 additional SNs (20\%). In total, 26 SNs were laparoscopically removed (range, 1-4 per patient).

Histopathologic examination revealed metastases in 1 excised SN alongside the testicular vessels. This patient was treated with 4 cycles of carboplatin, etoposide, and bleomycin chemotherapy. All other patients were observed in a surveillance program. During a median follow-up of $21 \mathrm{mo}$ (range, 2-50 mo), no recurrences developed in the 9 patients in whom the SN was negative for metastasis.

\section{DISCUSSION}

This study demonstrates the ability of SPECT/CT to provide preoperative anatomic localization of retroperitoneal SNs in patients with stage I testicular cancer. Furthermore, it demonstrates the feasibility and additional
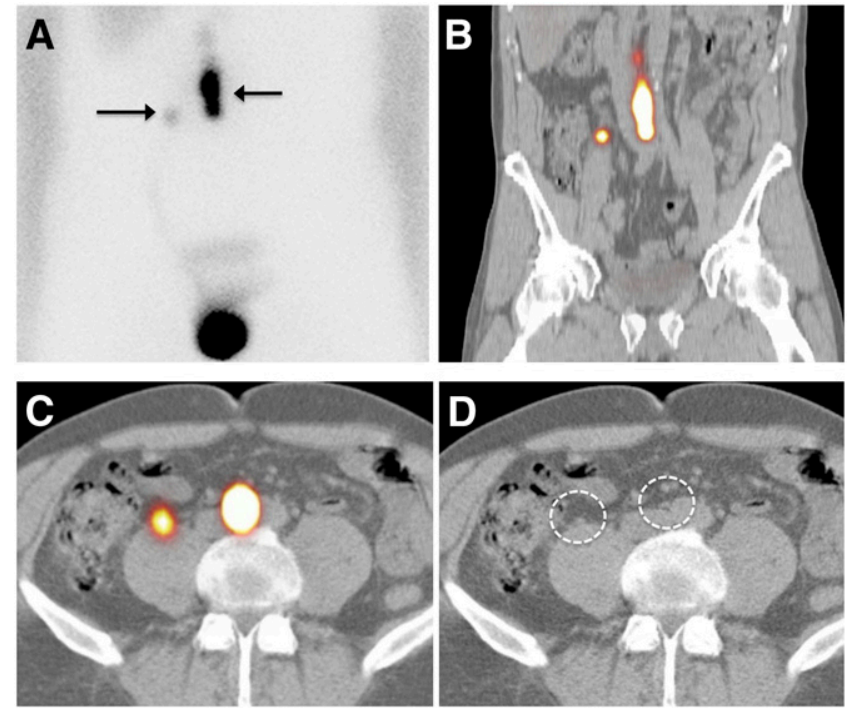

FIGURE 2. (A) Planar anterior image showing drainage from right testicle to 2 adjacent lymph nodes in medial area of abdomen. Some uptake is also seen in more laterally located lymph node (arrows). (B) Coronal fused SPECT/CT image showing both medial lymph nodes between aorta and vena cava and lateral node in trajectory of right testicular vessels. (C) Transverse SPECT/CT image showing 2 SNs displayed in yellow. (D) CT image showing that medial SN corresponds to small lymph node ventral to area between aorta and cava, whereas lateral node is in area ventral to psoas muscle (circles). In this funicular SN, micrometastases were found at histopathology.

value of the use of a portable $\gamma$-camera for real-time intraoperative $\mathrm{SN}$ visualization.

The route of administration of ${ }^{99 \mathrm{~m}} \mathrm{Tc}$-nanocolloid has previously been studied (6). In that study, funicular administration showed lymph node uptake only in the inguinal region, which does not reflect testicular tumor drainage, and intratesticular administration resulted in retroperitoneal SN visualization, in accordance with known drainage patterns. In addition, that study performed preoperative lymphatic mapping using planar lymphoscintigraphy only. Planar lymphoscintigraphy images are not able to visualize SNs in relation to the surrounding anatomic structures. This aspect remains crucial when laparoscopy is used to remove SNs located in retroperitoneal areas. In the current study, SPECT/CT visualized SNs in their anatomic habitat in paracaval, interaortocaval, and paraaortic regions in all patients. Moreover, retroperitoneal SNs along the testicular vessels, an uncommon area of lymphatic drainage in testicular cancer, were found in 3 patients (9). Their position was accurately indicated by preoperative SPECT/CT but not by conventional lymphoscintigraphy (Fig. 2). In 1 of these nodes, micrometastases were found. Previous reports on the use of SPECT/CT in SN detection also showed favorable results using this modality in other malignancies (10). Sequential planar images remain essential to distinguish between first- and second-echelon nodes.

Accurate staging with SN biopsy can be achieved only if all nodes on a direct drainage pathway from the tumor are 

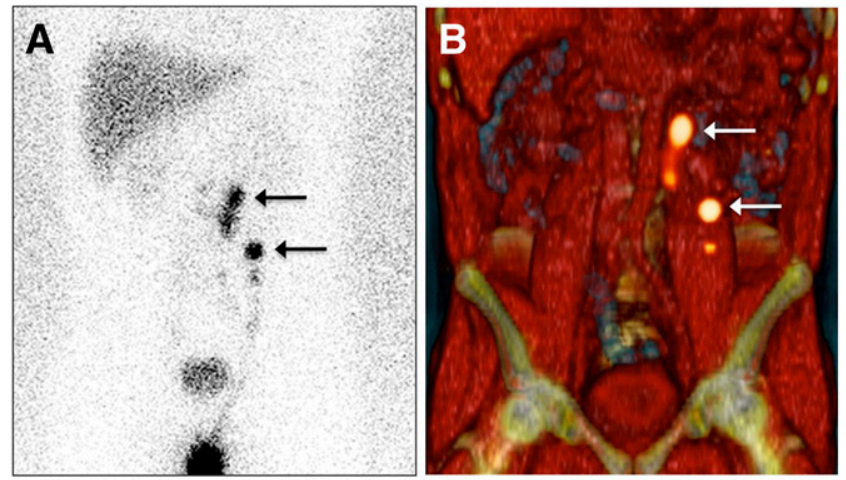

$\gamma$-probe. The latter does not always detect remaining nodes deep in the retroperitoneum because it is difficult to position the tip at the correct angle. We expect that with increasing experience, these aspects will shorten the procedure and increase its reliability.

The refinement of the SN procedure may enable better selection of patients who would benefit from adjuvant treatment after orchidectomy, avoiding costly surveillance and decreasing the risk of finding lymph node metastases at a later stage. Further study is required to substantiate the clinical value of the $\mathrm{SN}$ procedure in this disease.
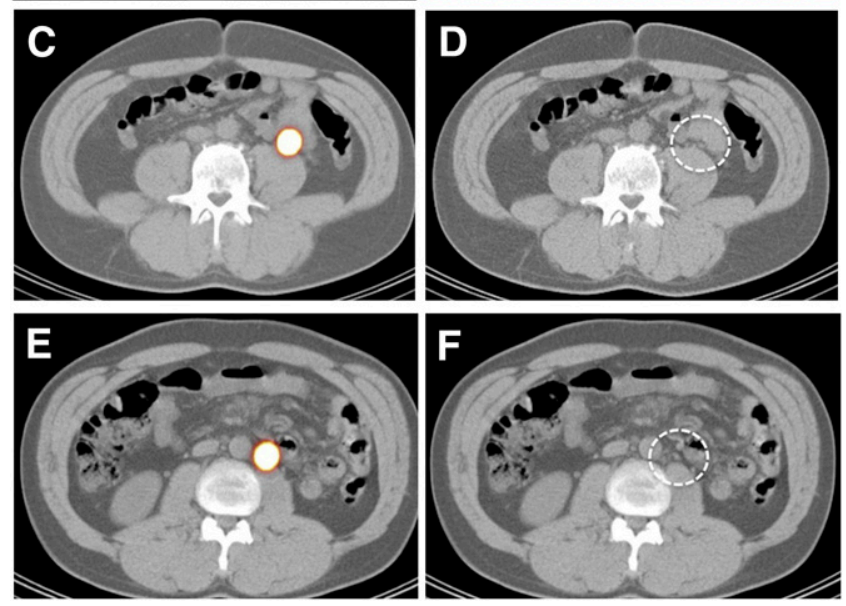

FIGURE 3. (A) Planar anterior image showing drainage from injection site in left testicle to 2 abdominal lymph nodes (arrows) and radioactivity along lymphatic channel indicating lymphatic tract visualization. (B) Fused SPECT/CT image displayed with 3-dimensional volume rendering showing cranial lymph node alongside aorta and caudal node in retroperitoneal trajectory of left testicular vessels (arrows). Some remaining activity is seen in lymphatic channels. (C) Transversal fused SPECT/CT image showing caudal SN (displayed in yellow). (D) CT image showing corresponding lymph node ventral from left psoas muscle (within circle). (E and F) Paraaortic SN is displayed in both fused SPECT/CT (yellow on E) image and CT (within circle on F) image.

identified and harvested. The procedure in the operation room is facilitated by the anatomic information preoperatively given by SPECT/CT. A portable $\gamma$-camera was introduced and evaluated as an additional tool to improve intraoperative SN retrieval in laparoscopic procedures for urologic malignancies. A ${ }^{125}$ I seed pointer was introduced to further facilitate intraoperative SN localization (8). The use of this device enables real-time display and the combination of visual and acoustic signals to find the site of the SN. This protocol provides good results in terms of laparoscopic $\mathrm{SN}$ detection rates $(100 \%)$. The portable $\gamma$-camera can detect residual radioactivity caused by other SNs after removal of the initial SN at the same site. Such adjacent additional SNs are not depicted separately on the SPECT/CT images. This feature resulted in the removal of $20 \%$ additional SNs in these series. The intraoperative camera increased the certainty about the completeness of the surgical procedure and complements the laparoscopic

\section{CONCLUSION}

SPECT/CT enables anatomic localization of retroperitoneal SNs in patients with testicular cancer. In addition, realtime image guidance using a portable $\gamma$-camera together with a laparoscopic $\gamma$-ray detection probe improves intraoperative SN detection and appears to identify $20 \%$ additional SNs.

\section{DISCLOSURE STATEMENT}

The costs of publication of this article were defrayed in part by the payment of page charges. Therefore, and solely to indicate this fact, this article is hereby marked "advertisement" in accordance with 18 USC section 1734.

\section{ACKNOWLEDGMENTS}

We thank the urologists, surgical assistants, and technicians of the nuclear medicine department for their contribution.

\section{REFERENCES}

1. Stephenson A, Klein E. Surgical management of low-stage nonseminomatous germ cell testicular cancer. BJU Int. 2009;104:1362-1368.

2. de Wit R, Fizazi K. Controversies in the management of clinical stage I testis cancer. J Clin Oncol. 2006;24:5482-5492.

3. Powles T, Robinson D, Shamash J, Moller H, Tranter N, Oliver T. The long-term risks of adjuvant carboplatin treatment for stage I seminoma of the testis. Ann Oncol. 2008;19:443-447.

4. Stephenson AJ. Current treatment options for clinical stage I seminoma. World $J$ Urol. 2009;27:427-432.

5. Cummins S, Yau T, Huddart R, Dearnaley D, Horwich A. Surveillance in stage I seminoma patients: a long-term assessment. Eur Urol. 2010;57:673-678.

6. Tanis PJ, Horenblas S, Valdés Olmos RA, Hoefnagel CA, Nieweg OE. Feasibility of sentinel node lymphoscintigraphy in stage I testicular cancer. Eur J Nucl Med Mol Imaging. 2002;29:670-673.

7. Vermeeren L, Meinhardt W, Bex A, et al. Paraaortic sentinel lymph nodes: toward optimal detection and intraoperative localization using SPECT/CT and intraoperative real-time imaging. J Nucl Med. 2010;51:376-382.

8. Vermeeren L, Valdés Olmos RA, Meinhardt W, et al. Intraoperative radioguidance with a portable gamma camera: a novel technique for laparoscopic sentinel node localisation in urological malignancies. Eur J Nucl Med Mol Imaging. 2009;36:1029-1036.

9. Weissbach L, Boedefeld EA. Localization of solitary and multiple metastases in stage II nonseminomatous testis tumor as basis for a modified staging lymph node dissection in stage I. J Urol. 1987;138:77-82.

10. Vermeeren L, van der Ploeg IMC, Olmos RAV, et al. SPECT/CT for preoperative sentinel node localization. J Surg Oncol. 2010;101:184-190. 